\title{
AUTOMATIC TEXT SUMMARIZATION USING SEMANTICALLY EXTENDED WORD POSITION INFORMATION
}

\author{
Koji Samejima, Noriko Matsumoto and Norihiko Yoshida \\ Department of Information and Computer Sciences, Saitama University \\ Saitama 338-8570, Japan
}

\begin{abstract}
Many automatic text summarization services use extractive methods, which pick up sentences according to their importance out of a given document. The importance is estimated using several metrics such as position information and semantic information. Most methods combine these metrics using weighted linear summation. This paper proposes more fine-grained integration of position information and semantic information to achieve better estimation of importance, which leads to better quality of summaries.
\end{abstract}

\section{KEYWORDS}

Extractive Summarization, Position and Semantic Information, WordNet

\section{INTRODUCTION}

Recently, there is an increasing demand for technologies to enable efficient grasp of the document contents for small screen devices. In particular, automatic text summarization technologies have been attracting attentions and a lot of studies have been done. It is because the Internet and mobile devices have become easily accessible to the large amount of digital data.

Many practical systems and researches make summaries using extractive methods. An extractive method generates summaries extracting highly important parts out of a given document. The manner how to evaluate importance affects the quality of summaries.

There have been many studies to apply artificial intelligence techniques to automatic text summarization: deep learning [Singh, S. P. et al., 2016], reinforcement learning [Lee, G. H. and Lee, K. J., 2017], and genetic algorithms [Meena, Y. K. and Gopalani, D., 2015] for example. However, such AI techniques are computationally intensive, and techniques based on text features are still important and effective, because such conventional techniques are comparatively lightweight.

There are various features for evaluating the importance. In many cases, one feature is used to evaluate one importance. In this paper, we focus on combination of two features of semantic information and position information. Position information is shallow but easy to handle. Semantic information is deep but difficult in the sense that it requires thesaurus to analyze. When evaluating the importance from two features, single importance is calculated from each feature first, and then combined importance is calculated as a weighted linear sum of the both importance. However, in this method, importance is calculated individually by each feature. This is not a method that takes into account of multiple features.

Here we propose a new method that combines semantic information and position information. It is a semantically extended word position information as a new feature combining semantic information and position information. The word position information is a method of calculating the word importance metrics according to their appearance order. In addition, to examine the semantic information we use the electronic lexical database WordNet [Miller, G. A., 1995] [Fellbaum, C., 1998].

The rest of the paper is organized as follows: Section 2 describes the current situation and related studies on automatic summarization. Section 3 explains the details of our proposed method. Section 4 describes our experiments and evaluation results. Section 5 discusses conclusions and our future work. 


\section{AUTOMATIC SUMMARIZATION}

Automatic summarization is a technology that summarize the contents of the original document to make short concise document automatically. Automatic summarization began as one of the natural language processing technologies in the 1950s. Automatic summarization methods can be divided into two: single document summaries and multiple document summaries. Methods to make summaries can be categorized into two types: abstractive methods and extractive methods [Das, D. and Martins, A. F. T., 2007].

The abstractive methods analyze original documents using language analysis techniques to identify key contents in the document, and generates summaries using natural language generation techniques. It is necessary to use complex and sophisticated techniques. Therefore, it is technically difficult. In addition, the methods construct summaries from the scratch, therefore it is difficult to make readable sentences.

In the extractive methods, importance is calculated for words, sentences and paragraphs. After evaluating the importance, a summary is generated extracting parts out of a given document according to the importance metrics. It is less likely that sentences in the summary are not readable. Consequently, most practical automatic summarization systems use extractive methods rather than the abstractive methods.

In the extractive method, summary sentences would be different depending on the manner to evaluate the importance. In other words, the method to evaluate importance affects the accuracy and quality of the summary. Therefore, various features to evaluate importance have been proposed. The extractive method evaluates importance using features one or more, to produce a summary.

In this section we summarize features to evaluate importance of parts and integration methods of features.

\subsection{Term Frequency}

Words for important concepts tend to appear frequently in a document. A word that represents an important concept in the document is considered to appear frequently in the document. Based on this assumption, a term frequency feature gives high importance to frequent words. The term frequency feature is well used to find important words in a document. There are TF and TF*IDF methods using the term frequency feature. TF (term frequency) raises word importance in proportion to the frequency. TF*IDF (inverse document frequency) raises word importance that appear most in a particular document than words appearing in common in many documents.

\subsection{Clue Expression}

Clue expression refers to an expression which is a clue to identify the location of a key sentences in the document. In a document, there are some parts that represent its subject (in-text summary). Some expressions, such as "summary", "In this document", and "In short", imply clues which literally identify in-text summaries, i.e., important sentences. On the contrary, there are negative clue expressions which imply unimportant parts. Expressions which begin with such as "for example" are considered to be a negative clue expression.

\subsection{Semantic Information}

Summarization that uses semantic information has appeared in recent years. To use semantic information, a thesaurus is necessary to examine semantic connections and relationships among words. WordNet, which is an electronic lexical database developed at Princeton University, is one of famous English thesauri. In WordNet, words that represent the same concept are classified to the same group called synset (synonym set). Most synsets are connected with other synsets according to semantic relationships. The semantic relationships of WordNet include "synonyms", "antonyms", "hypernyms", and "holonyms". Using the WordNet, it is possible to evaluate semantic connections and relationships among words.

There are some instances of automatic summarization using lexical chains for semantic information. A lexical chain is a graph of words with a lexical connection. [Barzilay, R. and Michael, E., 1999] created lexical chains of documents giving the appropriate concept on WordNet against noun or compound nouns in the document. Furthermore, in order to evaluate the strength of the lexical chain, they introduced visualize lexical chain and manually ranked lexical chains. Based on manually ranked data, they defined the 
expression which express a strong lexical chain. Finally, they extracted sentence which was associated to strong lexical chains to make summaries.

[Allahyari, M. et al., 2017] classified types of lexical links in a document (forward link, backward link, highly related link, medium related link, and low related links). In addition, they generated summaries by giving importance depending on the characteristics of the word link.

Some researches for automatic summarization created clusters of concepts in WordNet. [Plaza, L. et al., 2010] transformed a document to a concept graph using WordNet. They created concept clusters by analyzing concept graphs. A concept cluster represented a set of important concepts in a given document. They created summaries by evaluating an importance metric of each sentence based on the similarity between the clusters.

\subsection{Position Information}

Position information evaluates importance according to the structure of sentences. There are some common patterns for positions where significant sentences and words appear. For example, the heading of a document briefly represents contents of the document itself, therefore words in the heading are considered important. In the case of a document in English, the first sentence in a paragraph often represents the contents of the paragraph. In the case of newspaper and news articles, sentences at the beginning briefly summarize the contents of an article as a lead. A lead method makes a summary by extracting lead sentences.

[Brandow, R. et al., 1995] compared summaries of newspapers and magazines between summaries generated by a system called ANES (Automatic News Extraction System) and summaries generated by a simple lead method. ANES was a summarization system using the term frequency and heading information. The results indicated that the lead method is more acceptable than ANES at $74 \%$ to $92 \%$. The lead method is a simple technique, however it is useful for newspaper and magazine articles because the lead of these articles contain many important sentences.

[Ouyang, Y. et al., 2010] conducted a study to compare the word position information and sentence position information. They compared the performance of two summarization systems, one used sentence position information and the other used word position information. The result of the comparison indicated generally that word position system was better.

[Armano, G. et al., 2012] used position information such as headlines and first and last paragraphs to make summaries. They utilized summaries to select web advertisements. They found that it is possible to increase the amount of information in a summary when adding header information to the summary. In addition, they revealed that the summary containing headline and first-last paragraphs contained the most amount of information.

\subsection{Feature Integration}

There are various features to evaluate importance as described before. However, when we use various features, it is necessary to calculate a single importance by integrating importance calculated on various features. The simplest way to integrate features is to use a weighted linear sum as shown in the equation below, where $f$ stands for feature, $w_{f}$ stands for weight of feature $f$, and $i_{f}$ stands for importance that are calculated on feature $f$.

$$
\text { importance }=\sum_{f} w_{f} \times i_{f}
$$

There is a method of using a machine learning approach when evaluating the importance by integrating the multiple features. [Neto, J. L. et al, 2002] integrated multiple features such as position information and term frequency by machine learning. They used two learning algorithms, C4.5 is a classifier using a decision tree and a naive Bayes classifier is a famous machine learning algorithm. Also they used hand-made summaries as references. They compared four summarization systems in the experiment, which are a system based on the naive Bayes classifier, one based on C4.5, one based on the lead method, and Microsoft WordSummarizer. As a result, the system using the naive Bayes classifier made the best summaries. Other three systems made summaries of almost the same quality. 


\section{PROPOSED METHOD}

In this section, we explain our proposed method WPxS. WPxS is a method using semantically extended word position information. First, we describe the method using term frequency (TF) and method using word position information (WP). Then, we explain how to evaluate the importance at each methods using an example sentence below:

boy boy child parent parent boy

\subsection{TF (Term Frequency)}

This method evaluates the word importance using the term frequency. In the case of the example sentence, freq $($ boy $)=3$, freq $($ child $)=1$, and freq $($ parent $)=2$, where freq $(w)$ stands for frequency of word $w$. The importance of each word is shown below. A word appearing more frequently is given a higher importance.

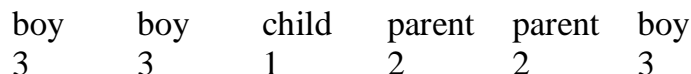

\subsection{WP (Word Position)}

This method evaluates the word importance according to the appearance order of each word. In our proposed method, first, we give the word importance by TF. Then we adjust importance according to the appearance order. The method for adjusting importance in the word position information is as follows: a word appearing earlier is given higher importance. We adjust the importance by the inverse of the appearance order.

In the case of the example sentence, word "boy" appears three times. The first "boy" gets impo $($ boy 1$)=\operatorname{freq}($ boy $) / 1$, the second "boy" gets impo(boy 2$)=f r e q($ boy $) / 2$, and the third "boy" gets $\operatorname{impo}($ boy3 $)=\operatorname{freq}($ boy $) / 3$, where $\operatorname{impo}(w)$ stands for word importance $w$. Importance of a word is $\operatorname{impo}(w)=\operatorname{freq}(w) / \operatorname{order}(w)$, where $\operatorname{order}(w)$ stands for the appearance order of word $w$. The importance of each word is shown below.

$\begin{array}{llllll}\text { boy } & \text { boy } & \text { child } & \text { parent } & \text { parent } & \text { boy } \\ 3 & 1.5 & 1 & 2 & 1 & 1\end{array}$

\subsection{WPxS (Word Position and Synonym)}

The proposed method WPxS is a technique that combines the word position information (WP) and the synonym information (S). This means that WPxS augments the word position information with semantic information. When evaluating the word importance metrics with TF and WP, the importance metric is evaluated word by word. However, in a document, the same concept is sometimes represented by different words. In this method, we put together a word that has the same concept to synonym set using an electronic lexical database WordNet. Summaries are made by analyzing the term frequency and word position in the synonym set unit rather than the word unit.

In the case of the example sentence, "boy" and "child" are synonyms. Therefore, the method put together "boy" and "child" to one of the synonyms set "S1". In this case, freq $($ S1 $)=$ freq $($ boy $)+$ freq $($ child $)=4$. The first "S1" importance is impo(S11) = freq $(S 1) / 1$, the second "S1" importance is impo(S12) $=$ freq $(S 1) / 2$, the third "S1" importance is impo(S13) = freq $(S 1) / 3$, and the forth "S1" importance is impo $(S 14)=$ freq (S1)/4. The importance of each word is shown below. In this method TF and WP are applied in synonym set unit.

$\begin{array}{llllll}\text { boy } & \text { boy } & \text { child } & \text { parent } & \text { parent } & \text { boy } \\ 4 & 2 & 1.33 & 2 & 1 & 1\end{array}$




\subsection{WPxS2}

WPxS2 defines synonyms of synonyms as one of the synonyms set, while WPxS defines synonyms individually. In other words, WPxS2 makes summaries by examining synonym relationships more deeply. In the case of the example sentence, WPxS2 gives the same result as WPxS. However, in the longer document, the result may be different.

\section{EVALUATION}

\subsection{System Outline}

The outline of the automatic summarization system which we implemented is as follows.

1) Input Document

The system is given a plain text file written in English as a document.

2) Preprocessing

The system does some preprocessing before summarization. It splits the document in a sentence or word units in order to analyze the sentences and words in the document. The system removes unnecessary symbols such as brackets and operators, and removes unnecessary words such as preposition, conjunctions and articles. In addition, the system calculates the frequency and the appearance order of words.

3) Synonyms Analysis

The system examines the synonym relationships between each word using WordNet. It puts together the words that have the same concept to the synset.

4) Sentence Scoring

The system evaluates the importance metric of each word based on the results of the previous processes (word frequency, the appearance order and synonym relationship). The importance metric of the sentence is calculated using the equation below, where $s$ stands for a sentence.

$$
\operatorname{impo}(s)=\sum_{i}^{n} i m p o\left(w_{i}\right) \quad s=\left\{w_{1}, w_{2}, \cdots, w_{n}\right\}
$$

\section{5) Output Summary}

The system generates a summary extracting statements in the descending order of the importance metric, no longer than 500 characters.

\subsection{Experimental Setup}

We have prepared a dataset for automatic summarization assessment, Opinosis Opinion Dataset 1.0 [Ganesan, K. et al., 2010]. This dataset is the set of manually-made reference summaries and 51 documents. There are three to five reference summaries in one document. In this experiment, we use 10 pairs of documents and reference summaries to evaluate summarization system performance.

In addition, we use the automatic evaluation measure of ROUGE [Lin, C.-Y., 2004] for evaluation of the automatic summarization. ROUGE evaluates the performance of automatic summarization by comparing the reference summary and the machine-generated summary. There are various evaluation method in ROUGE. For example, ROUGE-N compares the n-gram, and ROUGE-S compares skip-by-gram. In this experiment, we evaluate the effectiveness of the proposed method using ROUGE-L, which compares the longest common subsequence. 
In the experiment, four systems generate summaries for the 10 documents. Summarization systems to be compared are TF, WP, WP\$xS, and WpxS2.

\subsection{Experiment Results}

The experimental result is shown in Table 1 regarding the average score of the four summarization systems for 10 documents. This score was calculated by ROUGE-L comparing the reference summaries and machine summaries. WPxS2 obtains the highest score. From the results, extending the word position information by using semantic information was proven to make more accurate summaries.

Table 1. Average score of 10 documents

\begin{tabular}{lllll}
\hline System & TF & WF & WPxS & WPxS2 \\
\hline Average R-L & 0.380 & 0.442 & 0.448 & 0.450 \\
\hline
\end{tabular}

Next, in order to show the performance of the system vs. the length of the documents. We evaluated each system using four documents with different lengths. The results are shown in Figure 1. The horizontal axis represents the four documents that are used in the evaluation, where the numbers in square brackets represent the number of characters in each document. The vertical axis represents the score when evaluated each system by ROUGE-L. The larger the value is, the better the summary gets. In the experiment result, TF gives the best score at doc [9573]. Conversely, TF gives the worst score at doc [14965], and doc [25725]. The score of WP is low at doc [30903], while the scores of WPxS and WPxS2 using synonym approach are high. From these experimental results, the systems using the synonym information considered particularly effective in the long document. This must be because a long document contains various kind of words and synonym set.

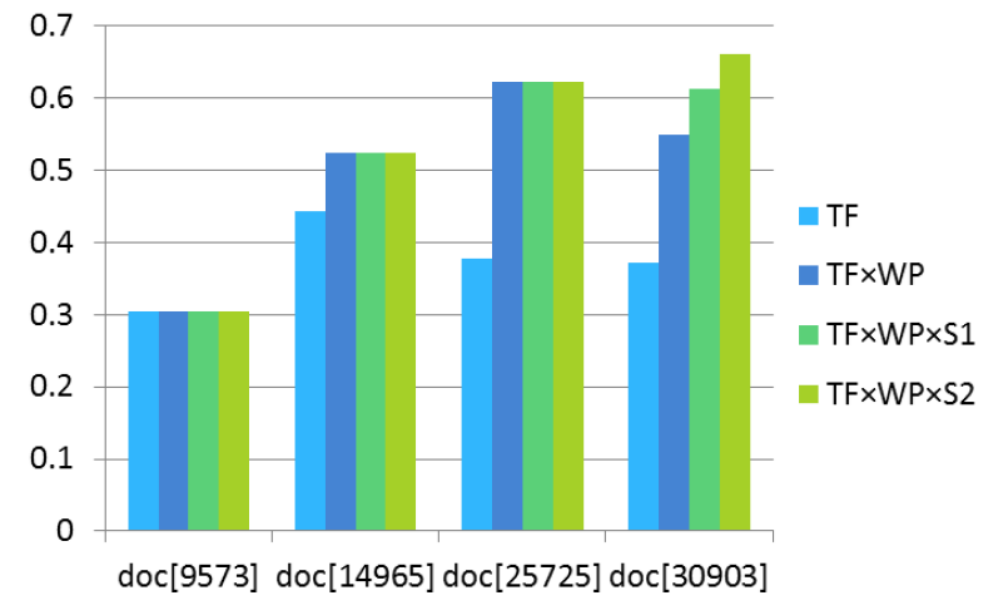

Figure 1. Effects of the document lengths.

\section{CONCLUSION AND FUTURE WORK}

In this paper, we presented a new feature that combined the semantic information and location information, i.e. semantically expanded word position information. we performed some experiments to evaluate performance of the system. We used summary evaluation dataset called Opinosis Opinion Dataset 1.0 and the ROUGE summary automatic evaluation scales in order to confirm the effectiveness of the proposed method in the experiments. In comparative experiments, we confirmed that our proposed method is effective particularly for long documents. 
Our future work includes handling of composite words. In this study, we do not take into account the composite words. If we handle the composite words, it is possible to use more words in the synonym relationships.

\section{REFERENCES}

Allahyari, M. et al., 2017. Text Summarization Techniques: A Brief Survey. arXiv, 1707.02268v3, 9 pages.

Armano, G. et al., 2012. Experimenting Text Summarization Techniques for Contextual Advertising. Proc. Second Italian Workshop on Information Retrieval, pp. 27-28.

Barzilay, R. and Michael, E., 1999. Using Lexical Chains for Text Summarization, Advances in Automatic Text Summarization, MIT Press, pp.111-121.

Brandow, R. et al., 1995. Automatic Condensation of Electronic Publications by Sentence Selection, Information Processing and Management, Vol.31, No.5, pp. 675-685.

Das, D. and Martins, A. F. T., 2007. A survey on Automatic Text Summarization. Literature Survey for the Language and Statistics II course at CMU, pp. 192-195.

Fellbaum, C., 1998. WordNet: An Electronic Lexical Database, MIT Press.

Ganesan, K. et al., 2010. Opinosis: A Graph Based Approach to Abstractive Summarization of Highly Redundant Opinions, Proc. 23rd Int. Conf. on Computational Linguistics, pp. 340-348.

Lee, G. H. and Lee, K. J., 2017. Automatic Text Summarization Using Reinforcement Learning with Embedding Features. Proc. 8th Int. Joint Conf. on Natural Language Processing, pp. 193-197.

Lin, C.-Y., 2004. Rouge: A Package for Automatic Evaluation of Summaries. Proc. Workshop on Text Summarization Branches Out, pp. 74-81.

Meena, Y. K. and Gopalani, D., 2015. Evolutionary Algorithms for Extractive Automatic Text Summarization. Procedia Computer Science, Vol.48, Elsevier, pp. 244-249.

Miller, G. A., 1995. WordNet: A Lexical Database for English. Comm. ACM, Vol.38, No.11, pp. 39-41.

Neto, J. L. et al, 2002. Automatic Text Summarization using a Machine Learning Approach. Advances in Artificial Intell., Springer, pp. 205-215.

Ouyang, Y. et al., 2010. A Study on Position Information in Document Summarization. Proc. 23rd Int. Conf. on Computational Linguistics, pp. 919-927.

Plaza, L. et al., 2010. Automatic Summarization of News Using Wordnet Concept Graphs. Int. J. on Computer Science and Information System, Vol.5, No.1, pp. 45-57.

Singh, S. P. et al., 2016. Bilingual Automatic Text Summarization Using Unsupervised Deep Learning. Proc. 2016 Int. Conf. on Electrical, Electronics, and Optimization Techniques, pp. 1195-1200. 